\title{
Rehabilitation With Implant Supported Fixed Prosthesis- A Case Report
}

\author{
Reena Luthra* and Poonam Pathania
}

Department of Prosthodontics, Swami Devi Dyal Dental College \& Hospital, Barwala, Panchkula, Haryana, India

*Corresponding author: Reena Luthra, Department of Prosthodontics, Swami Devi Dyal Dental College \& Hospital, Barwala, Panchkula, Haryana, India, Tel: +919815998430; E-mail: harshtanya@yahoo.co.in

Received date: January 17, 2018; Accepted date: January 29, 2018; Published date: February 07, 2018

Copyright: @ 2018 Luthra R, et al. This is an open-access article distributed under the terms of the Creative Commons Attribution License, which permits unrestricted use, distribution, and reproduction in any medium, provided the original author and source are credited.

Citation: Luthra R, Pathania P (2018) Rehabilitation With Implant Supported Fixed Prosthesis- A Case Report. Eur Exp Biol Vol. 8 No. 1:4.

\section{Abstract}

Treatment of the edentulous patient with fixed reconstructions using multiple implants in both dental arches has many benefits compared to a conventional removable denture, including increased patient satisfaction, improved speech, aesthetics, function and self esteem. With a continuing increase in the use of implants, a significant percentage of edentulous population is benefitting from the advantages of fixed implant restorations. The choice of a suitable prosthesis for a specific case is determined to a great extent by the underlying residual bone volume as well as the teeth being replaced. The aim of the present case report is to describe the oral rehabilitation of a patient with a fixed implantsupported prosthesis.

Keywords: Full-arch prosthesis; Implant prosthesis; Fixed implant reconstruction

\section{Introduction}

The use of dental implants to replace lost teeth in edentulous patients has become a popular mode of treatment in recent years. The social embarrassment caused by moving dentures and the constant attempts to stabilize them have led many patients to seek for implant supported fixed restorations. Fixed implant restorations are totally implant supported, with no transfer of load to denture bearing areas, thus avoiding the possibility of further resorption associated with tissue-borne prostheses. Several studies indicate that implant supported restorations performed using meticulous surgical and restorative skills can provide long-lasting benefits to edentulous patients [1]. However, the clinician may have to face numerous challenges in accomplishing the task [2,3]. Failure to understand stress factors and stress distribution can lead to bone loss and restoration failure [4]. Thorough pre operative treatment planning, restorative driven surgery and splitting the framework in two or three parts can control the distribution of forces [4-7]. This article explains in detail the treatment planning and surgical and prosthetic steps taken to rehabilitate a patient with edentulous lower jaw and few natural teeth in upper jaw with implant restorations.

\section{Case Report}

A 62-year-old male patient visited the prosthodontic clinic with several decayed and mobile teeth in both arches. The patient had no systemic illness. Patient was reluctant to wear removable dentures and wanted fixed teeth. He was educated about the treatment option of having multiple implants to support fixed restorations. The surgical and the prosthetic procedures were discussed in detail and he gave his consent for a definitive implant-supported fixed prosthesis.

Preoperative surgical evaluation included a clinical examination and panoramic radiograph (Figure 1). After a detailed clinical evaluation, it was decided to extract all remaining mandibular and maxillary teeth with the exception of maxillary incisors and right maxillary canine as they were periodontally healthy and patient had a strong desire to retain them. The diagnostic mounting was carried out. Conventional procedures were followed to fabricate interim removable dentures. The dentures were duplicated and radiographic templates were prepared. Cone beam computerized tomography (CBCT) scan showed adequate height and width of bone at all implant sites. The CBCT information was formatted in software for interactive CT imaging. Provisional virtual implant simulation was performed as per the available bone height and width, along the long axis of the alveolar ridge.

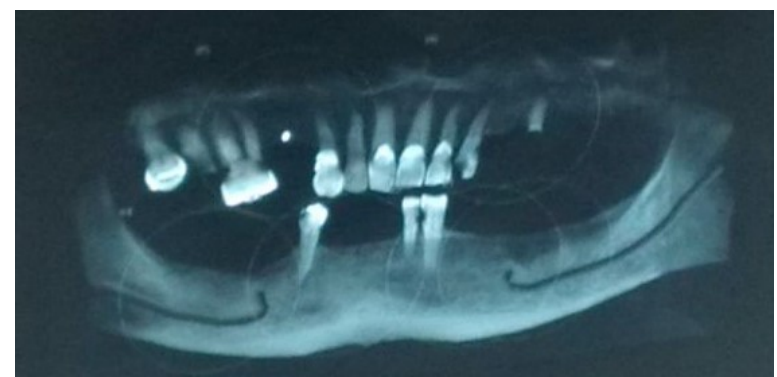

Figure 1: Pre-operative OPG.

Patient was pre-medicated with appropriate oral antibiotics and analgesics. Local anaesthetic was administered and mucoperiosteal flaps were raised. Three threaded external hex design implants (BioHorizons, BioHorizons, Inc, Birmingham, 
Alabama) were positioned in pre-selected positions in the maxillary right quadrant and four implants in maxillary left quadrant. In the mandible, seven implants were positioned in bilateral canine, second premolar and first molar positions and one in incisor region. The implant placement was strategically decided to avoid cantilevers in final prostheses.

The implants were submerged below the crestal bone level. A two-stage technique was used and cover screws were placed. Suturing was carried out in interrupted horizontal mattress pattern. The sutures were removed after seven days and the removable dentures were relined with soft denture liner (Permasoft, Dentsply, USA) to avoid creating pressure on the implants or the mucosa. Soft diet was recommended to avoid excessive loading of implants during the six months healing period.

Prosthetic phase: Six months later, patient was called for the second stage surgery and prosthetic phase. The implant sites were completely healed and free of any signs of inflammation. Incisions were given and the cover screws were removed. Transmucosal healing abutments were placed in all the implants.

The healing abutments were removed after 2 weeks when the gingival collars were formed around the implants and the sites were prepared for impression. Closed tray impression technique was followed using special trays obtained from the diagnostic cast. Ball top impression copings were attached to the implants. A panoramic radiograph was made to confirm the complete seating of impression copings. Closed tray impressions were made with addition silicone impression material (Aquasil, Dentsply).

The impressions were removed and the implant copings were placed in their respective positions in the impressions (Figure 2). Implant analogs were attached to the impression copings and casts were poured. The abutments were attached to the analogs on the cast and jigs were fabricated for trial (Figure 3). The jig trial was done to ascertain the accuracy of the impression. Jigs of both the arches were tightened in the patient's mouth and a radiograph was obtained to ensure a complete and passive seating (Figure 4).

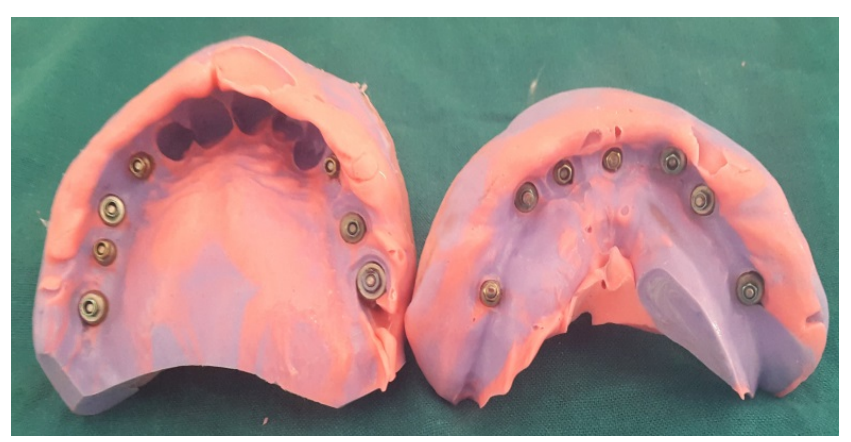

Figure 2: Impressions.

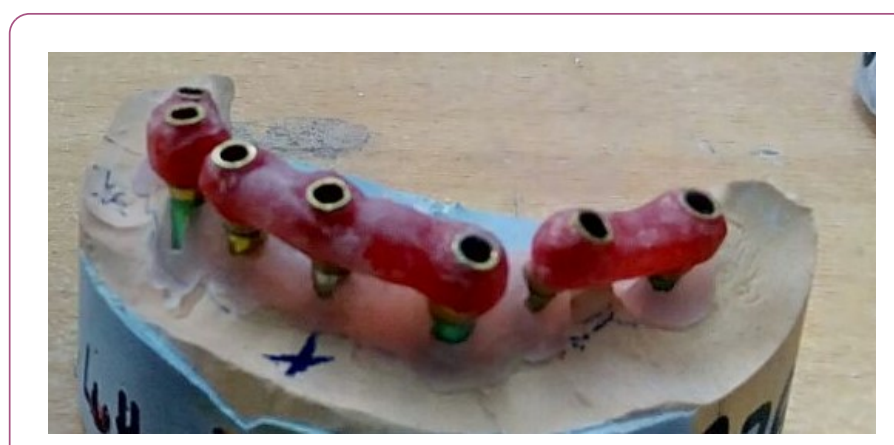

Figure 3: Verification jig mandible.

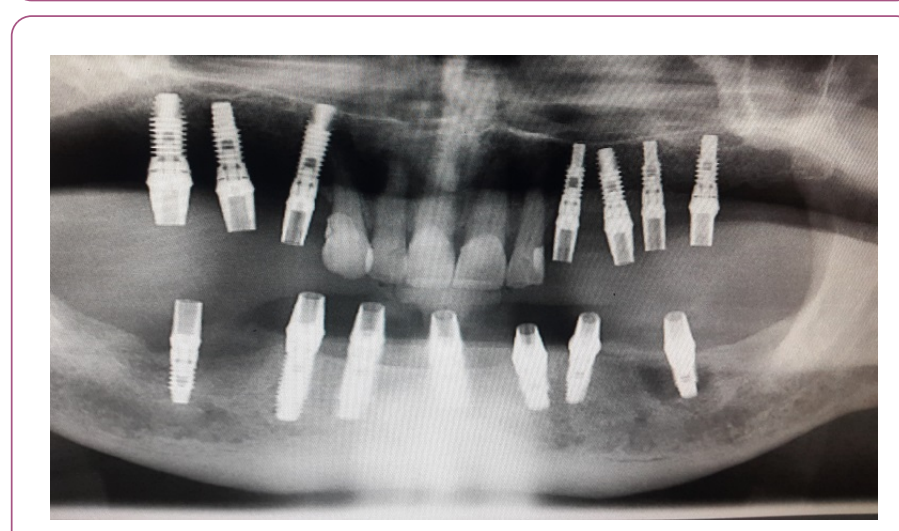

Figure 4: OPG with jig trial in place.

Customized trial denture bases were fabricated on the casts to record the maxillomandibular relations and were stabilized intraorally with light body addition silicone impression material. The maxillary cast was mounted on a semi-adjustable articulator using a face-bow. Vertical jaw relations were recorded to allow sufficient space for the final prostheses. The mandibular cast was then mounted using a centric relation record on wax occlusal rims.

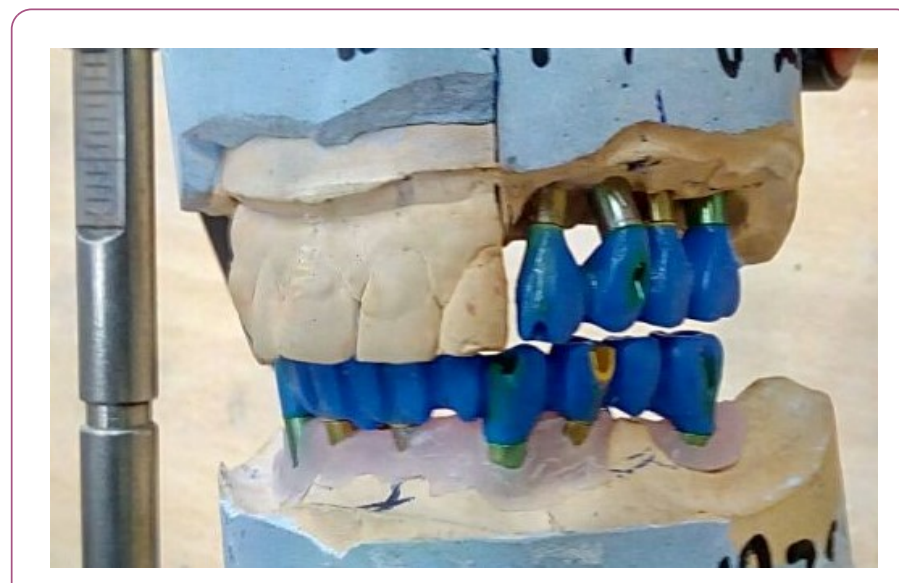

Figure 5: Wax pattern fabrication (left side).

Thereafter, wax patterns for the metal frameworks were fabricated (Figures 5-7). Segmented prosthetic designs were used. A split was planned on the right side between mandibular 
canine and premolar to compensate for mandibular flexure (Figure 7).

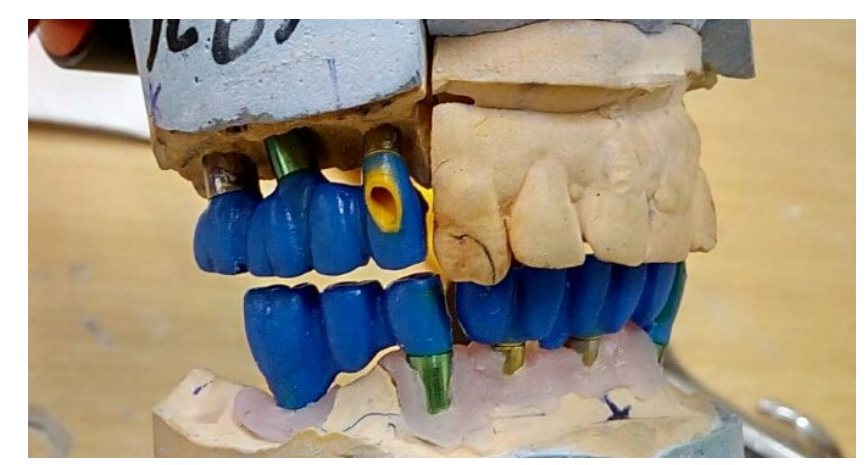

Figure 6: Wax pattern fabrication (right side).

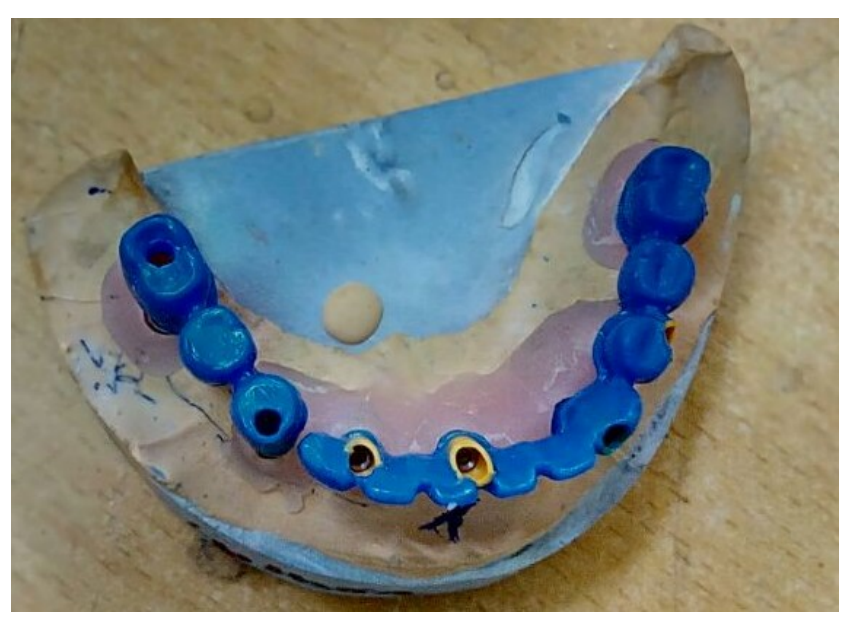

Figure 7: Wax pattern fabrication (mandible occlusal view).

A screw retained prostheses design was selected to allow easy retrievability and maintenance. The implant orientation at certain locations was such that the retaining screws would pass through the facial aspect of the dental prosthesis (Figures $\mathbf{5}$ and 6). The frameworks or substructure were cast and then evaluated clinically. The screws were tightened sequentially ensuring a passive fit. $A$ radiograph was taken to confirm the complete seating of the framework. A new centric relation record was made using Alu-Wax as an inter-occlusal bite registration material (Figure 8). The metal frameworks were returned to the laboratory for porcelain veneering. (Ivoclar Vivadent, Germany). The metal ceramic restorations were fixed on the implants, and occlusal adjustments were performed with articulating paper of thickness $25 \mu \mathrm{m}$ to eliminate all interferences [8-10]. A canine guided occlusion with posterior disclusion during excursions was provided. After glazing, final tightening was done with the recommended torque (Figure 9). The screw access holes were sealed with gutta percha and resin composite. Defective restorations in the natural anterior teeth were also replaced. The patient was comfortable and aesthetically satisfied after insertion. Oral hygiene instructions were reinforced. Patient was put on a regular recall i.e. after 24 hours, 1 week, 1 month, and 1 year. Patient did not report any complications in the recall visits.

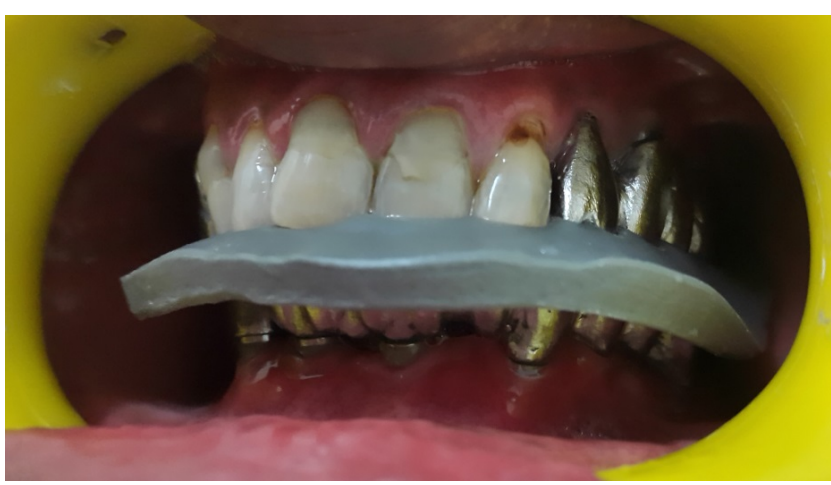

Figure 8: Interocclusal record in centric relation.

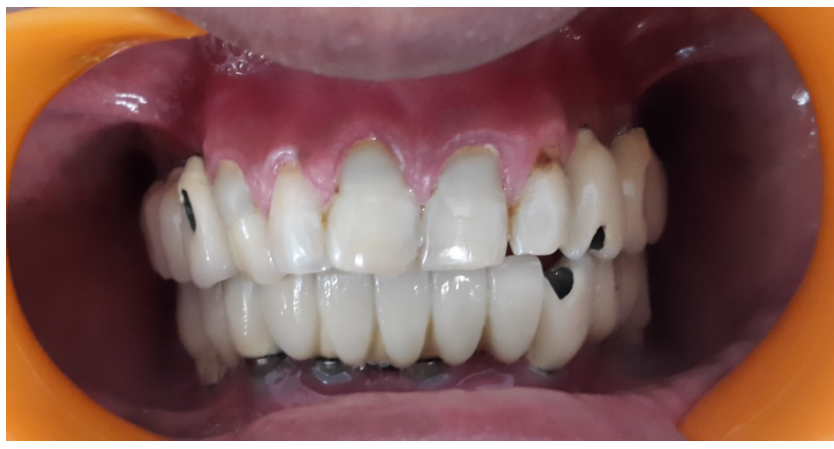

Figure 9: Metal-ceramic prostheses tightened into implants.

\section{Discussion}

Traditionally, four to five implants in the mandible and five to six implants in the maxilla with distal cantilevers has been a popular approach among the implant clinicians [11-17]. From the biomechanical point of view, this classical protocol may not appropriately address the complex requirements of edentulism. The literature recommends a minimum of four implants for a fixed restoration but more implants should always be placed for biomechanical advantages and to avoid cantilevers. An increase in the antero-posterior spread and more number of supporting implants increase the predictability of a successful outcome.

Current research recommends one implant for each missing tooth. Therefore, in the present case, seven implants were placed in maxilla, one for each missing tooth. In mandible also, seven implants were placed in well spread positions. The implant placement was strategically decided to avoid cantilevers in final prostheses, in contrast to the protocol used in other dental practices $[15,16]$.

In case of mandibular full arch fixed prosthesis, flexure of mandible may create stresses on the implants posterior to the interforaminal region [8-10]. Splitting the framework near the region of mental foramen can help to allow the flexure of mandible without causing undue forces to the implants. Some people prefer a split in the superstructure at symphsis región 
[16]. In the present case, instead of fabricating the popular onepiece restoration [14], a split was planned on the right side between mandibular canine and premolar to compensate for mandibular flexure. Advantages of a segmented prosthesis are stress distribution and that the loss of one implant may not result in loss of the entire reconstruction.

The critical performance of the screw joint is affected by the handling of the screw seat. Discrepancy between the screw seat and the retentive screw may lead to early screw loosening.

Although immediate loading is being more admired by patients and restorative dentists alike, literature cautions at several places that chances of failure are heightened in cases of immediate replacements. Therefore, a two stage surgical technique was followed in this case.

Cement retained implant restorations are gaining reputation as they are simple, esthetic and economical. But such restorations are difficult to retrieve and any residual cement in the soft tissues surrounding the implant may lead to periimplant disease [18]. A screw retained prostheses design was selected to allow easy retrievability and maintenance.

\section{Conclusion}

Implant supported fixed restorations can serve as an excellent treatment modality for edentulous patients. The prosthetic therapeutic success requires a detailed pre-surgical analysis based on prosthetically driven implant position, judicious selection of prosthetic materials, prosthesis design and proper maintenance with a rational understanding of patient expectations and limitations.

\section{References}

1. Ganeles J, Rosenberg MM, Holt RL, Reichman LH (2001) Immediate loading of implants with fixed restorations in the completely edentulous mandible: report of 27 patients from a private practice. Int J Oral Maxillofac Implants 16: 418-426.

2. Göthberg C, Bergendal T, Magnusson T (2003) Complications After Treatment with Implant-Supported Fixed Prostheses: A Retrospective Study. Int J Prosthodont 16: 201-207.

3. Bragger $\mathrm{U}$, Aeschlimann S, Burgin W, Hammerle CHF, Lang NP (2001) Biological and technical complications and failures with fixed partial dentures (FPD) on implants and teeth after four to five years of function. Clin Oral Impl Res 12: 26-34.

4. Marin DOM, Dias KC, Paleari AG, Pero AC, Filho JNA, et al. (2015) Split-Framework in Mandibular Implant-Supported Prosthesis. Case Reports in Dentistry 2015: 502394.
5. Misch CE (2008) Contemporary Implant Dentistry. Missouri: 3rd ed. Elsevier Mosby Publication; 2008.

6. Gallucci GO, Avrampou M, Taylor JC, Elpers J, Thalji G, et al. (2016) Maxillary Implant-Supported Fixed Prosthesis: A Survey of Reviews and Key Variables for Treatment Planning. Int J Oral Maxillofac Implants 31(suppl): s192-s197.

7. Al-Taweel SM (2015) Controversy about implant number and distribution in full mouth implant-supported fixed prosthesis: A case report. J Int Oral Health 7: 126-129.

8. Breeding LC, Dixon DL, Sadler JP, McKay ML (1995) Mechanical considerations for the implant tooth-supported fixed partial denture. J Prosthet Dent 74: 487-492.

9. Becker CM, Kaiser DA, Jones JD (2000) Guidelines for splinting implants. J Prosthet Dent 84: 210-214.

10. Cakan U, Anil N, Aslan Y (2006) Prosthetic Rehabilitation of a mandibular gunshot defect with an implant supported fixed partial denture: a clinical report. J Prosthet Dent 95: 274-279.

11. Ogino Y, Kihara M, Yamada J, Toriya K, Koyano K (2015) Implant treatments for edentulous maxilla with anterior hyperfunction. Journal of Oral Implantology 41: 731-735.

12. Mertens C, Steveling HG (2011) Implant-supported fixed prostheses in the edentulous maxilla: 8-year prospective results. Clin Oral Impl Res 22: 464-472.

13. Sunil M, Reddy BM, Reddy TS, Reddy NR (2013) Full mouth rehabilitation with fixed implant-supported prosthesis: A case report. J NTR Univ Health Sci 2: 292-295.

14. Rajgiri SU, Dayalan M (2016) Full mouth rehabilitation with implant supported fixed prosthesis. Int J Oral Implantol Clin Res 7: 73-80.

15. Çötert HS, Zeytinoğlu B, Zeytinoğlu M (2014) Mock-Up Driven Designing of Full-Mouth Implant-Supported Metal-ceramic Fixed Prostheses. Dentistry 4: 204.

16. Mathew M, John B, George A (2013) An early loaded implantsupported mandibular complete arch fixed prosthesis in a young completely edentulous patient: A case report. Journal of Oral Implantology 39:487-495.

17. Bencharit S, Sacco DS, Border MB, Barbaro CP (2010) Full mouth rehabilitation with implant-supported prostheses for severe periodontitis: A Case Report. Open Dent J 4: 165-171.

18. Wilson TG (2009) The positive relationship between excess cement and peri-implant disease: A prospective clinical endoscopic study. J Periodontol 80: 1388-1392. 